\title{
Reverse-Remodeling Effects of Angiotensin II Type 1 Receptor Blocker in a Canine Atrial Fibrillation Model
}

\author{
Hideko Nakashima, MD*; Koichiro Kumagai, MD*
}

\begin{abstract}
Background The reverse-remodeling effect of angiotensin II type 1 receptor blocker (ARB) on atrial fibrillation $(\mathrm{AF})$ is unclear.

Methods and Results Sustained AF was induced in 20 dogs by 4-week rapid atrial pacing. The AF duration, atrial effective refractory period (AERP) and intra-atrial conduction time (CT) were measured every 2 weeks. After 4-week pacing, dogs were randomly assigned to control $(n=10)$ and ARB (olmesartan; $n=10)$ groups. Olmesartan was administered orally $\left(3 \mathrm{mg} \cdot \mathrm{kg}^{-1} \cdot \mathrm{day}^{-1}\right)$ after pacing was terminated, and continued for the 4-week recovery period. After 4-week pacing, AERP shortening, CT prolongation and AF maintenance were not significantly different between the 2 groups. During the recovery, AERP recovered to baseline in both groups. CT remained prolonged in the control group during the recovery, but recovered to baseline in the olmesartan group. The mean AF duration in the olmesartan group after 4-week-recovery was significantly shorter than that in the control group $(58 \pm 20$ vs $1,337 \pm 226 \mathrm{~s}, \mathrm{p}<0.001)$. Olmesartan significantly decreased interstitial fibrosis compared with the control group ( $9 \pm 1 \%$ vs $15 \pm 1$ at the right atrial appendage, $\mathrm{p}<0.001)$.

Conclusion Olmesartan has a reverse-remodeling effect on AF-induced structural changes, indicating that it may be useful for preventing AF recurrence after the termination of sustained AF. (Circ J 2007; 71: 1977-1982)
\end{abstract}

Key Words: Angiotensin; Atrial fibrillation; Electrophysiology

$A$ rial fibrillation (AF) is one of the most common arrhythmias, but is still difficult to treat because of its complicated electrophysiological and pathological properties! AF itself causes electrical and structural changes, which are called electrical and structural remodeling, and these changes are favorable for the maintenance of AF $2-6$ Previous studies have shown that structural abnormalities can induce conduction heterogeneity, which promotes local reentry and leads to the development of AF, ,4,6-8 Furthermore, some studies have demonstrated that although the electrical changes induced by AF rapidly recover after conversion to sinus rhythm (SR), the structural abnormalities persist and are vulnerable to AF induction.,9 We previously reported that an angiotensin II type 1 receptor blocker (ARB) can help prevent the promotion of AF by suppressing the development of an arrhythmogenic structural substrate ${ }^{10}$ However, the reverse-remodeling effect of ARB on existing AF-induced structural changes after the termination of $\mathrm{AF}$ is unclear. The purpose of this study was to investigate whether ARB therapy can help to reverse AFinduced structural remodeling and suppress AF after SR is restored in a canine atrial pacing model.

(Received April 24, 2007; revised manuscript received July 11, 2007; accepted August 7, 2007)

Department of Cardiology, Fukuoka University, Fukuoka, Japan

*Both authors contributed equally to this work. No conflict of interest exists. No disclosure exists.

Mailing address: Koichiro Kumagai, MD, Department of Cardiology, Fukuoka University, 7-45-1 Nanakuma, Jonan-ku, Fukuoka 814-0180,

Japan._E-mail: kxk@fukuoka-u.ac.jp

\section{Methods}

Animal Preparation and Groups

All experiments were performed in accordance with the guidelines specified by the Institutional Animal Care and Use Committee, the American Heart Association Policy on Research Animal Use, and the Public Health Service Policy on the Use of Laboratory Animals.

Ten mongrel dogs of either sex, weighing $24-26 \mathrm{~kg}$, were randomly divided into 2 groups. In the olmesartan group $(\mathrm{n}=10)$, oral administration of olmesartan $\left(3 \mathrm{mg} \cdot \mathrm{kg}^{-1} \cdot \mathrm{day}^{-1}\right.$ [Sankyo Co Ltd, Tokyo, Japan]) was started after rapid atrial pacing was terminated, and was continued for a 4week recovery period. The dogs in the control group $(\mathrm{n}=10)$ did not receive olmesartan.

All dogs were anesthetized with an intravenous injection of pentobarbital $(25 \mathrm{mg} / \mathrm{kg})$, and, after intubation and mechanical ventilation, anesthesia was maintained with halothane. A tachy-pacing generator (Medtronic Co Ltd, Minneapolis, MN, USA) was implanted in a subcutaneous pocket in the neck and attached to a pacing lead in the right atrial appendage (RAA). The chest was opened through the right fourth intercostal space, and 4 electrode pairs were sutured to 4 sites in the right atrium (appendage, and high lateral, low lateral and anterior wall). When surgery was completed, the dogs were given antibiotics and then allowed to recover. Postoperative care included the administration of antibiotics and analgesics.

\section{Experimental Protocol}

Baseline Electrophysiological Study One week after the operation, the dogs were re-anesthetized with pentobarbital $(25 \mathrm{mg} / \mathrm{kg})$ and ventilated with halothane. The arterial 
Table 1 Hemodynamic Parameters in the Control and Olmesartan Groups

\begin{tabular}{|c|c|c|c|c|c|c|}
\hline & \multicolumn{3}{|c|}{$\overline{~ C o n t r o l}$} & \multicolumn{3}{|c|}{ Olmesartan } \\
\hline & Baseline & Pace- $4 w$ & Recovery- $4 w$ & Baseline & Pace- $4 w$ & Recovery- $4 w$ \\
\hline$S B P$ & $112 \pm 4$ & $109 \pm 5$ & $113 \pm 4$ & $116 \pm 4$ & $113 \pm 4$ & $109 \pm 4$ \\
\hline$D B P$ & $66 \pm 2$ & $59 \pm 3$ & $65 \pm 2$ & $63 \pm 2$ & $58 \pm 2$ & $61 \pm 2$ \\
\hline$P C W P$ & $8 \pm 1$ & $12 \pm 1^{\S}$ & $9 \pm 1$ & $8 \pm 1$ & $13 \pm 1^{\S}$ & $8 \pm 1$ \\
\hline Mean PAP & $10 \pm 1$ & $17 \pm 1 *$ & $13 \pm 1$ & $11 \pm 1$ & $16 \pm 1 *$ & $12 \pm 1$ \\
\hline$R A P$ & $5 \pm 1$ & $8 \pm 1 *$ & $6 \pm 1$ & $5 \pm 1$ & $8 \pm 1 *$ & $5 \pm 1$ \\
\hline
\end{tabular}

$4 w, 4$ weeks; SBP, systolic blood pressure; DBP, diastolic blood pressure; PCWP, pulmonary capillary wedge pressure; PAP, pulmonary artery pressure; RAP, right atrial pressure.

${ }^{*} p<0.001,{ }_{s} p<0.01$ compared with the baseline.

blood gases were adjusted to a $\mathrm{pH}$ of between 7.35 and 7.45 during ventilation. The surface ECG lead II, intracardiac electrograms and blood pressure were continuously monitored and recorded during the experiment. The pulmonary capillary wedge pressure, the pulmonary artery pressure and the right atrial pressure were measured using a Swan-Ganz catheter.

Atrial effective refractory periods (AERPs) at 4 sites were measured at 3 basic cycle lengths (BCLs) $(200,300$, $400 \mathrm{~ms}$ ). Five basic drive stimuli were followed by a single premature stimulus, and all stimuli were twice the diastolic threshold. The S1-S2 interval was increased in steps of $2 \mathrm{~ms}$, and AERP was determined to be the shortest S1-S2 interval that resulted in a propagated atrial response. AERP dispersion was measured as the difference between the maximum and minimum AERP among 4 sites. Intra-atrial conduction times (CT) from the RAA to the other 3 sites (high lateral, low lateral and anterior wall) were measured during appendage pacing at each $\mathrm{BCL}$, and $\mathrm{CT}$ dispersion was also measured among 3 CTs.

$\mathrm{AF}$ was defined as a rapid, irregular atrial rhythm with varying atrial electrogram morphology. The inducibility of $\mathrm{AF}$ was assessed by premature atrial stimulation during AERP measurement and atrial burst pacing $(10 \mathrm{~Hz}$ for $1-$ $10 \mathrm{~s})$. To estimate the mean AF duration, AF was induced 10 times if the $\mathrm{AF}$ duration was $<15 \mathrm{~min}$ and 5 times if $\mathrm{AF}$ lasted between 15 and $30 \mathrm{~min}$. AF $>15 \mathrm{~min}$ was considered sustained. If induced AF persisted $>30$ min, electrical cardioversion was performed. When electrocardioversion was applied, AF induction was repeated after a 15-min rest period.

Electrophysiological Study After Rapid Atrial Pacing After the measurement of baseline electrophysiological and hemodynamic parameters, programmed atrial pacing was started at 400 beats/min using 2-ms pulses at twice the threshold current and this was continued for 4 weeks in both the control and olmesartan groups. The tachy-pacing generator was turned off every 2 weeks to allow for repeated assessment by an electrophysiological study. After 4 weeks of rapid atrial pacing, the same electrophysiological protocol was performed every 2 weeks during the 4-week recovery period.

Histology At the end of the experiments, the heart was quickly removed and weighed. To investigate the influence of rapid pacing on pathological properties, the atrial tissues of 5 sham dogs without rapid atrial pacing were observed at the same time. The tissues of the left and right atrial free wall and appendages were cut into small blocks approximately $10 \times 5 \mathrm{~mm}$ and immersed in $10 \%$ phosphate-buffered formalin for $24 \mathrm{~h}$. After dehydration, each section was cut into $4 \mu \mathrm{m}$ thick slices. Deparaffinized sections were stained with hematoxylin-eosin and Masson's trichrome. Microscopic images were scanned into a personal computer with Photoshop and the image files were analyzed with NIH software. Connective tissue was differentiated on the basis of its color and expressed as a percentage of the reference tissue area. These analyses were performed by a pathologist who was unaware of the treatment.

\section{Statistical Analysis}

All of the data analyses were performed using Statistical Analysis System (SAS) Software Package (Ver.9.1, SAS Institute Inc, Cary, NC, USA) at Fukuoka University (Fukuoka, Japan). Changes in continuous variables in each group during the study period were examined by a repeated measures analysis of variance (ANOVA). Differences in continuous variables between 2 groups during the study were examined by ANOVA. Differences in continuous variables among the control, sham, and olmesartan groups were examined by ANOVA and the multiple comparison test of Scheffe. All values are expressed as mean \pm mean standard error. Significance was considered to be less than 0.05 unless indicated otherwise.

\section{Results}

\section{Hemodynamic Changes}

Hemodynamic data are shown in Table 1. There were no significant differences in the blood pressure, pulmonary capillary wedge pressure, mean pulmonary artery pressure, and mean right atrial pressure between the control and olmesartan groups throughout the experiment.

\section{Changes in Electrophysiological Properties}

In both the control and olmesartan groups, AERP shortening was observed after 2 weeks of rapid pacing, and continued during pacing (Fig 1). The degree of AERP shortening produced by 4 weeks of pacing was not significantly different between the 2 groups (Fig 1). The shortened AERP completely recovered to the baseline value in 2 weeks after rapid pacing was terminated in both groups (Fig 1). The time course of both AERP shortening and of recovery was similar between the 2 groups.

The intra-atrial CT from the RAA to the other 3 sites progressively increased during pacing, and the value after 4 weeks of pacing was significantly longer than the baseline in both groups (Fig 1). After pacing was terminated, CT prolongation gradually recovered in the olmesartan group, but remained prolonged in the control group during the recovery period, and its value after 4 weeks of recovery was significantly longer than that in the olmesartan group. The dispersion of CT also significantly increased after 4 weeks 
of pacing in both groups (control group: from $8.2 \pm 1.0$ to $13.1 \pm 0.8 \mathrm{~ms}, \mathrm{p}<0.05$; olmesartan group: from $8.1 \pm 0.9$ to $11.6 \pm 0.6 \mathrm{~ms}, \mathrm{p}<0.001$, at BCL $300 \mathrm{~ms})$. After 4 weeks of recovery, the value in the olmesartan group returned to the baseline $(10.3 \pm 0.6 \mathrm{~ms}$, NS vs baseline), but that in the control group did not $(13.7 \pm 0.9 \mathrm{~ms}, \mathrm{p}<0.01 \mathrm{vs}$ baseline $)$.

At baseline, sustained AF (>900s) was not induced in either the control or olmesartan group (control group: 38 6 s; olmesartan group: 41+5 s) (Fig 1). Rapid pacing caused a significant increase in the duration of AF compared with baseline in both groups (Fig 1). None of the dogs showed spontaneous AF when the pacemaker was turned off. After 4 weeks of rapid pacing, sustained AF was easily induced in all dogs in both groups, and electrical cardioversion was performed in all of them. During the recovery period, sustained AF was still inducible in 6 of $10 \mathrm{dogs}$ in the control group, but was not induced at all in the olmesartan group, and the mean duration of AF in the olmesartan group was significantly shorter than that in the control group (control group vs olmesartan group: $1,337 \pm 226$ vs $58 \pm 20$ s after 4 weeks of recovery, $\mathrm{p}<0.001$ ) (Fig 1).

\section{Pathological Examination}

There was no significant difference in the body weight or the heart weight/body weight index between the 2 groups after 4 weeks of recovery (Table 2). Pericardial inflammation, effusion, and hemorrhage were not observed in any dog. Representative histological sections from each group are shown in Fig 2. Upon gross inspection, atrial tissue from the sham dogs appeared normal. In contrast, atrial tissue from the control dogs showed a large amount of interstitial fibrosis distributed throughout the tissue. In addition, heterogeneity in the size and arrangement of atrial myocytes was found in these tissues. These pathological abnormal findings were attenuated in the olmesartan-treated dogs.

A quantitative analysis of fibrosis is shown in Fig 3. The percentage of fibrosis in all atrial regions in the olmesartan group was markedly lower than that in the control group $(9 \pm 1 \%$ vs $15 \pm 1 \%$ at the RAA, $\mathrm{p}<0.001)$, but was greater than that in the sham group $(9 \pm 1 \%$ vs $4 \pm 1 \%$ at the RAA, $\mathrm{p}<0.05)$.

\section{Discussion}

\section{Main Findings}

This study has demonstrated for the first time that blockade of angiotensin II has reverse-remodeling effects on the structural changes caused by long-lasting AF after conver-

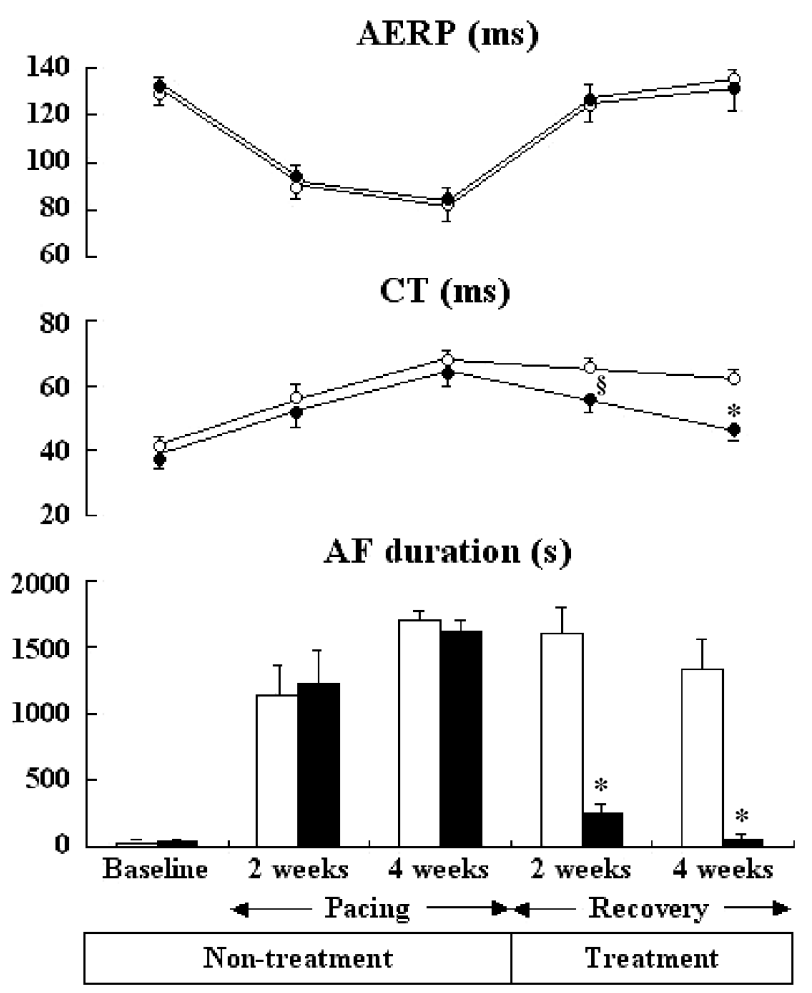

Fig 1. Time course of the atrial effective refractory period (AERP) of the right atrial appendage and intra-atrial conduction time (CT) from the right atrial appendage to the low right atrial wall at a basic cycle length of $300 \mathrm{~ms}$, and the mean duration of induced atrial fibrillation $(\mathrm{AF})$ in the control and olmesartan groups. The time course of AERP shortening and of recovery was similar between the 2 groups. The intra-atrial CT prolongation during rapid pacing was similar between the 2 groups. During the recovery period, the prolonged CT recovered in the olmesartan group, but not in the control group. The mean AF duration during rapid pacing was similar in the 2 groups, but that in the olmesartan group was significantly shorter than that in the control group during the recovery period. White circles and bars: control group; black circles and bars: olmesartan group. ${ }^{*} \mathrm{p}<0.001,{ }^{\S} \mathrm{p}<0.01$ compared with the control group.

Table 2 Characteristics After 4 Weeks of Recovery in the Control and Olmesartan Groups

\begin{tabular}{lcc}
\hline \hline & Control & Olmesartan \\
\hline Body weight $(\mathrm{kg})$ & $26 \pm 1$ & $25 \pm 2$ \\
Heart weight $(\mathrm{g})$ & $200 \pm 15$ & $182 \pm 10$ \\
Heart weight/body weight $(\mathrm{g} / \mathrm{kg})$ & $7.7 \pm 0.8$ & $7.3 \pm 0.3$ \\
\hline
\end{tabular}

A. Sham

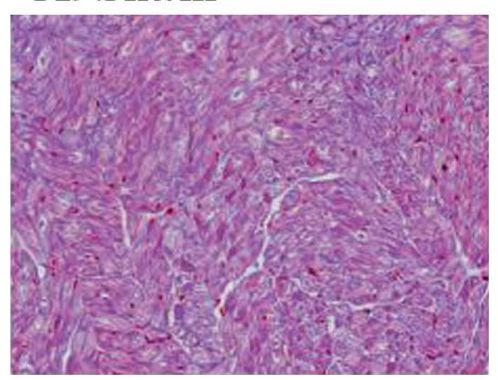

B. Control

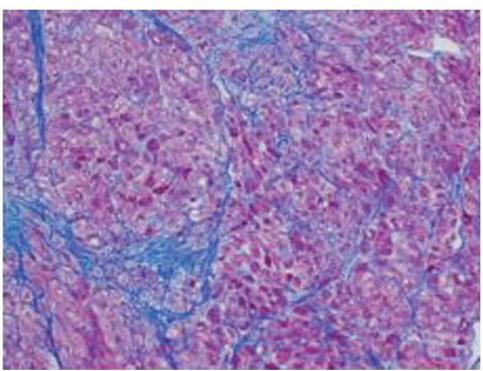

C. Olmesartan

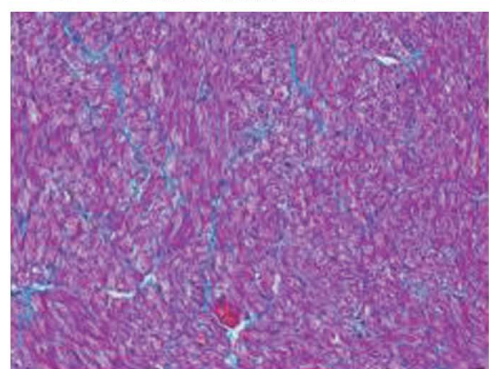

Fig 2. Representative histologic sections of the left atrial free wall from a sham dog (A), non-treated control dog (B), and olmesartan-treated $\operatorname{dog}(\mathrm{C})$. Atrial tissue from the sham dog appeared normal. Extensive interstitial fibrosis can be seen in the control dog, but is attenuated in the olmesartan group $(\times 200$; Masson trichrome stain). 


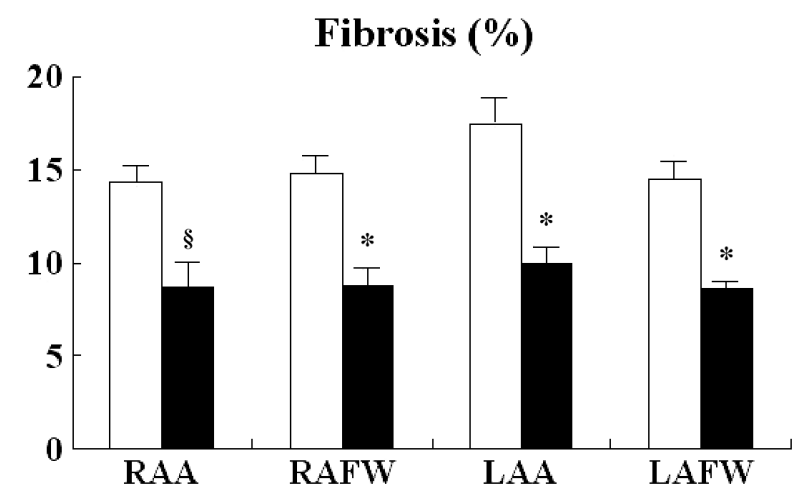

Fig 3. Percentage of fibrosis of the free walls and appendages in both atria after 4 weeks of recovery. The percentage of fibrosis in all atrial regions in the olmesartan group was lower than that in the control group. White bars: control group; black bars: olmesartan group. ${ }^{*} \mathrm{p}<0.001,{ }^{{ }} \mathrm{p}<0.01$ compared with the control group. RAA, right atrial appendage; RAFW, right atrial free wall; LAA, left atrial appendage; LAFW, left atrial free wall.

sion to SR. The major findings of this study are as follows: (1) the degree and time course of both AERP shortening and AERP dispersion were similar between the 2 groups throughout the experiment, (2) intra-atrial CT and CT dispersion were significantly increased during pacing in both groups; however, although CT and CT dispersion remained increased after the termination of pacing in the control group, olmesartan recovered these values to the baseline, (3) the mean duration of AF in the olmesartan group was significantly shorter than that in the control group, and (4) olmesartan significantly reduced the percentage of interstitial fibrosis compared with the control group. These findings indicate that AF vulnerability and its maintenance may be accounted for by the conduction delay and heterogeneity caused by the structural remodeling that remained, even after the restoration of SR, and that the blockade of angiotensin II may suppress not only AF promotion by preventing structural remodeling, but also AF recurrence, by improving the arrhythmogenic substrate.

\section{$A F$ and Reverse-Remodeling}

Previous instrumented animal experiments have shown that a shortened AERP and AF cycle length caused by longterm rapid atrial pacing ( $>3$ weeks) completely returned to the baseline levels by 1-2 weeks after the restoration of SR 3,9 Everett et $\mathrm{al}^{9}$ observed the time course of reverseremodeling during a follow-up period of 7-14 days after the termination of $>8$ weeks of rapid pacing in a canine model. They reported that despite complete reverse electrical remodeling, left atrial enlargement and cellular abnormalities remained after 7-14 days of SR? In that study, the occurrence of spontaneous $\mathrm{AF}$ and the frequency of premature atrial contractions tended to decrease with reverse electrical remodeling; however, the vulnerability to easily induced AF persisted in the setting of complete reverse electrical remodeling and continued anatomic abnormalities, suggesting that continued atrial anatomic abnormalities in response to chronic $\mathrm{AF}$ is the dominant factor in the recurrence of $\mathrm{AF}$, not electrical remodeling? Furthermore, Ausma et al reported that recovery from structural remodeling after 4 months of AF was a slow process and several structural changes were still present after 4 months of SR, and that induced AF episodes still occurred!1
In the present study, electrical remodeling and reverse electrical remodeling were similar to the results in those previous studies. Although AERP was completely normalized within 2 weeks of SR, atrial pathological abnormalities remained at 4 weeks after restoration to SR, and all animals in the control group had persisting vulnerability to AF induction. However, episodes of spontaneous AF and premature atrial contractions were not observed during 4 weeks of rapid pacing or during the recovery period, which might be because of the pacing duration, but based on the presence of AF vulnerability and structural abnormalities during a recovery period of 4 weeks after return to SR, 4 weeks of pacing was enough to evaluate the state of reverse-remodeling. In addition, progressive intra-atrial conduction delay occurred during pacing and continued for 4 weeks after the termination of pacing, which indicated that advanced structural changes, especially interstitial fibrosis, were not improved and remained for at least 4 weeks of observation. Therefore, it appears that persistent structural remodeling of the atria plays a critical role in maintaining conduction abnormality and AF vulnerability, and the reverse-remodeling of an arrhythmogenic substrate may be a therapeutic approach to suppressing recurrent $\mathrm{AF}$ after cardioversion.

\section{AngII Blockade and AF Reverse-Remodeling}

Recent clinical trials have reported that angiotensinconverting enzyme inhibitors (ACEI) and ARBs reduce the incidence of new-onset AF in patients with left ventricular dysfunction and congestive heart failure ${ }^{12-17}$ Animal experimental studies have also demonstrated that these drugs help to prevent $\mathrm{AF}_{10}^{10}$ and several possible mechanisms of these beneficial effects have been suggested: improved hemodynamics ${ }^{10}$ reduced atrial stretching ${ }^{18}$ modulation of ion channel function, ${ }^{19}$ and suppressed angiotensin II-induced atrial fibrosis 10 However, in addition to this suppression of new-onset AF, the AFFIRM study demonstrated that ACEI treatment suppressed AF relapse in patients with congestive heart failure among patients who had been randomized to rhythm control? 20 Furthermore, previous clinical studies demonstrated that patients who had been treated with amiodarone plus ACEI or ARB had a lower rate of recurrence of $\mathrm{AF}$ than patients treated with amiodarone alone after electrical cardioversion in patients with persistent $\mathrm{AF}^{21}$ In addition, Zaman et al demonstrated abbreviated signal-averaged $\mathrm{P}$-wave duration, reflecting improved atrial conduction abnormality, after successful electrical cardioversion in AF patients on long-term (6-12 months) ACEI therapy?2 Based on these results, angiotensin II inhibition may help to facilitate the process of reverse AF-induced arrhythmogenic remodeling and the subsequent long-term maintenance of SR by suppressing AF relapse after the cardioversion of persistent $\mathrm{AF}$. The previous clinical trials demonstrated that treatment with an ACEI or ARB had beneficial effects in AF patients with cardiac risk factors; ${ }^{12-17,22,23}$ however, the detailed mechanism of the effect of angiotensin II blockade on AF reverse-remodeling has not been clarified. In the present study, although the elevated atrial pressure was normalized in the both groups, sustained AF was still inducible only in the non-treated control group during the recovery period of 4 weeks, but was no longer inducible in the olmesartantreated group. Previously, it was reported that administration of losartan inhibited the stimulation of collagenase activity and reversed myocardial fibrosis in spontaneously hypertensive rats ${ }^{24}$ Furthermore, in a clinical study, the ARB, losartan, reversed myocardial fibrosis as the result of 
normalization of collagen type I metabolism in hypertensive patients.5 Thus, the inhibitory effects of olmesartan on AF vulnerability may represent a direct action against the process of atrial reverse-remodeling, not a secondary effect caused by improved atrial stretch.

\section{Study Limitations}

Electrophysiological parameters were measured only in the right atrium, and therefore electrophysiological characteristics in other areas are still unknown. However, in previous experiments, the time course and magnitude of both electrical and structural remodeling were similar between the right and left atrium, $4,5,9-11$ and in the present study olmesartan had equal reverse structural remodeling effects in both atria, indicating that melioration of conduction abnormalities might be observed in both atria in a parallel fashion.

Previous clinical studies have observed the presence of left ventricular diastolic dysfunction even in patients with isolated $\mathrm{AF}$, and demonstrated reverse morphological remodeling of the left atrium and improvement of left ventricular diastolic and systolic functions after restoration of SR by ablation for isolated $\mathrm{AF}^{26}$ Moreover, Ausma et al have reported that the recovery of atrial mechanical function after the restoration of SR is a slow process and related to the duration of AF before cardioversion!1,27 In the present study, although we did not assess atrial and ventricular function with echocardiography, there was no significant change in the time course or magnitude of the hemodynamic parameters measured using a Swan-Ganz catheter between the 2 groups. Thus, it is possible that olmesartan might have the beneficial effects on cardiac systolic and diastolic functions.

\section{Clinical Implications}

It is well known that treatment with both ACEI and ARB is effective for reducing risk factors for AF and AF-related complications $!^{12-17,22} \mathrm{~A}$ recent mega-analysis demonstrated that treatment with ACEI or ARB had beneficial effects on the inhibition of both new-onset of AF and AF relapse after cardioversion in patients with risk factors for $\mathrm{AF}$ (eg, congestive heart failure, hypertension with left ventricular hypertrophy)! ${ }^{12-17,22}$ We previously reported that ARB prevents the structural remodeling that promotes $\mathrm{AF}$ in a canine model, and in the present study, we showed that ARB has the potential to reverse AF-induced structural remodeling and suppress AF recurrence after cardioversion. Thus, ARB may be a novel therapeutic approach to the prevention of $A F$ recurrence after the termination of sustained $\mathrm{AF}$ in the clinical setting.

\section{References}

1. Allessie MA, Boyden PA, Camm AJ, Kleber AG, Lab MJ, Legato MJ, et al. Pathophysiology and prevention of atrial fibrillation. Circulation 2001; 103: 769-777.

2. Allessie M, Ausma J, Schotten U. Electrical, contractile and structural remodeling during atrial fibrillation. Cardiovasc Res 2002; 54: $230-246$.

3. Wijffels MC, Kirchhof CJ, Dorland R, Allessie MA. Atrial fibrillation begets atrial fibrillation: A study in awake chronically instrumented goats. Circulation 1995; 92: 1954-1968.

4. Ausma J, Wijffels M, Thone F, Wouters L, Allessie M, Borgers M. Structural changes of atrial myocardium due to sustained atrial fibrillation in the goat. Circulation 1997; 96: 3157-3163.

5. Wijffels MC, Kirchhof CJ, Dorland R, Power J, Allessie MA. Electrical remodeling due to atrial fibrillation in chronically instrumented conscious goats: Roles of neurohumoral changes, ischemia, atrial stretch, and high rate of electrical activation. Circulation 1997; 96: $3710-3720$.

6. Verheule S, Wilson E, Everett T 4th, Shanbhag S, Golden C, Olgin J. Alterations in atrial electrophysiology and tissue structure in a canine model of chronic atrial dilatation due to mitral regurgitation. Circulation 2003; 107: 2615-2622.

7. Nakajima H, Nakajima HO, Salcher O, Dittie AS, Dembowsky K, Jing $\mathrm{S}$, et al. Atrial but not ventricular fibrosis in mice expressing a mutant transforming growth factor-beta(1) transgene in the heart. Circ Res 2000; 86: 571-579.

8. Verheule S, Sato T, Everett T 4th, Engle SK, Otten D, Rubart-von der Lohe $\mathrm{M}$, et al. Increased vulnerability to atrial fibrillation in transgenic mice with selective atrial fibrosis caused by overexpression of TGF-beta1. Circ Res 2004; 94: 1458-1465.

9. Everett TH 4th, Li H, Mangrum JM, McRury ID, Mitchell MA, Redick JA, et al. Electrical, morphological, and ultrastructural remodeling and reverse remodeling in a canine model of chronic atrial fibrillation. Circulation 2000; 102: 1454-1460.

10. Kumagai K, Nakashima H, Urata H, Gondo N, Arakawa K, Saku K. Effects of angiotensin II type 1 receptor antagonist on electrical and structural remodeling in atrial fibrillation. J Am Coll Cardiol 2003; 41: $2197-2204$.

11. Ausma J, van der Velden HM, Lenders MH, van Ankeren EP, Jongsma HJ, Ramaekers FC, et al. Reverse structural and gap-junctional remodeling after prolonged atrial fibrillation in the goat. Circulation 2003; 107: 2051-2058.

12. The CONSENSUS Trial Study Group. Effects of enalapril on mortality in severe congestive heart failure: Results of the Cooperative North Scandinavian Enalapril Survival Study (CONSENSUS). $N$ Engl J Med 1987; 316: 1429-1435.

13. Pedersen OD, Bagger H, Kober L, Torp-Pedersen C. Trandolapril reduces the incidence of atrial fibrillation after acute myocardial infarction in patients with left ventricular dysfunction. Circulation 1999; 100: $376-380$

14. Vermes E, Tardif JC, Bourassa MG, Racine N, Levesque S, White $\mathrm{M}$, et al. Enalapril decreases the incidence of atrial fibrillation in patients with left ventricular dysfunction: Insight from the Studies Of Left Ventricular Dysfunction (SOLVD) trials. Circulation 2003; 107: 2926-2931.

15. L'Allier PL, Ducharme A, Keller PF, Yu H, Guertin MC, Tardif JC. Angiotensin-converting enzyme inhibition in hypertensive patients is associated with a reduction in the occurrence of atrial fibrillation. J Am Coll Cardiol 2004; 44: 159-164.

16. Wachtell K, Lehto M, Gerdts E, Olsen MH, Hornestam B, Dahlof B, et al. Angiotensin II receptor blockade reduces new-onset atrial fibrillation and subsequent stroke compared to atenolol: The Losartan Intervention For End Point Reduction in Hypertension (LIFE) study. $J$ Am Coll Cardiol 2005; 45: 712-719.

17. Maggioni AP, Latini R, Carson PE, Singh SN, Barlera S, Glazer R, et al. Valsartan reduces the incidence of atrial fibrillation in patients with heart failure: Results from the Valsartan Heart Failure Trial (Val-HeFT). Am Heart J 2005; 149: 548-557.

18. Webster MW, Fitzpatrick MA, Nicholls MG, Ikram H, Wells JE. Effect of enalapril on ventricular arrhythmias in congestive heart failure. Am J Cardiol 1985; 56: 566-569.

19. Caballero R, Delpon E, Valenzuela C, Longobardo M, Tamargo J. Losartan and its metabolite E3174 modify cardiac delayed rectifier $\mathrm{K}(+)$ currents. Circulation 2000; 101: 1199-1205.

20. Murray KT, Rottman JN, Arbogast PG, Shemanski L, Primm RK, Campbell WB, et al. Inhibition of angiotensin II signaling and recurrence of atrial fibrillation in AFFIRM. Heart Rhythm 2004; 1: 669675.

21. Ueng KC, Tsai TP, Yu WC, Tsai CF, Lin MC, Chan KC, et al. Use of enalapril to facilitate sinus rhythm maintenance after external cardioversion of long-standing persistent atrial fibrillation: Results of a prospective and controlled study. Eur Heart J 2003; 24: 20902098.

22. Zaman AG, Kearney MT, Schecter C, Worthley SG, Nolan J. Angiotensin-converting enzyme inhibitors as adjunctive therapy in patients with persistent atrial fibrillation. Am Heart J 2004; 147: 823-827.

23. Hirayama Y, Atarashi H, Kobayashi Y, Horie T, Iwasaki Y, Maruyama M, et al. Angiotensin-converting enzyme inhibitor therapy inhibits the progression from paroxysmal atrial fibrillation to chronic atrial fibrillation. Circ J 2005; 69: 671-676.

24. Varo N, Iraburu MJ, Varela M, López B, Etayo JC, Díez J. Chronic AT(1) blockade stimulates extracellular collagen type I degradation and reverses myocardial fibrosis in spontaneously hypertensive rats. Hypertension 2000; 35: 1197-1202.

25. Díez J, Querejeta R, López B, González A, Larman M, Martínez Ubago JL. Losartan-dependent regression of myocardial fibrosis is 
associated with reduction of left ventricular chamber stiffness in hypertensive patients. Circulation 2002; 105: 2512-2517.

26. Reant $\mathrm{P}$, Lafitte S, Jais P, Serri K, Weerasooriya R, Hocini M, et al. Reverse remodeling of the left cardiac chambers after catheter ablation after 1 year in a series of patients with isolated atrial fibrillation.
Circulation 2005; 112: 2896-2903.

27. Manning WJ, Silverman DI, Katz SE, Riley MF, Come PC, Doherty $\mathrm{RM}$, et al. Impaired left atrial mechanical function after cardioversion: Relation to the duration of atrial fibrillation. $\mathrm{J} \mathrm{Am} \mathrm{Coll} \mathrm{Cardiol}$ 1994; 23: $1535-1540$. 